\title{
Correlation of Urine Albumin/Creatinine Ratio (UACR), High Sensitivity C-Reactive Protein (hsCRP) and N-Terminal Pro Brain Natriuretic Peptide (NT-proBNP) with Atherosclerosis (OxLDL) in Centrally Obese Men
}

\author{
Nancy Pakpahan ${ }^{1,2 *}$, Mansyur Arief ${ }^{1}$, and Ilhamjaya Patellongi ${ }^{1}$
}

\begin{abstract}
${ }^{1}$ Post Graduate Program in Clinical Biochemistry, Hasanuddin University, Jl. Perintis Kemerdekaan Kav.10, Makassar, Indonesia ${ }^{2}$ Prodia Clinical Laboratory, Jl. Cisangkuy No.2, Bandung 40114, Indonesia

*Correspondence: nancy_itb@yahoo.com
\end{abstract}

\section{Abstract}

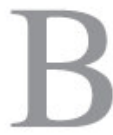

ACKGROUND: Obesity is closely associated with atherosclerosis risk and cardiovascular disease. Novel cardiovascular risk biomarkers such as Urine Albumin/Creatinine Ratio (UACR), High Sensitivity C-Reactive Protein (hsCRP) and N-Terminal pro Brain Natriuretic Peptide (NT-proBNP) have been observed to predict cardiovascular disease in the general population. The aim of this study was to observe the correlation of UACR, hsCRP and NT-proBNP with atherosclerosis (OxLDL) in centrally obese men.

METHODS: The study was observational with a cross sectional design done on 76 male subjects aged 30-50 years with central obesity and mean of age of 37 years. Urine albumin was determined by PEG enhanced immunoturbidimetric assay, urine creatinine by Jaffe without deproteinase, hsCRP by chemiluminescent immunometric assay, NT-proBNP by electrochemiluminescence (ECLIA) and OxLDL by ELISA.

RESULTS: There was significant correlation between hsCRP and OxLDL $(r=0.230, p=0.046)$. There was no significant correlation between UACR and OxLDL $(r=$ $-0.138, p=0.236$ ), neither between Log NT-proBNP and $\operatorname{OxLDL}(\mathrm{r}=-0.173, \mathrm{p}=0.136)$.
CONCLUSIONS: Atherosclerosis was significantly correlated with hsCRP (low grade inflammation).

KEYWORDS: NT-proBNP, UACR, hsCRP, OxLDL, atherosclerosis.

Indones Biomed J 2011; 3(1): 37-42

\section{Introduction}

Obesity has become a problem in all developed and developing countries. Unhealthy feeding pattern (high lipid and cholesterol with low dietary fiber) and less physical activity can be the cause of obesity. According to the International Diabetes Federation 2005, of more than 1,1 billion people worldwide, about 320 million have the problem of obesity (1).

Obesity is associated closely with atherosclerosis risk and cardiovascular disease. The development of obesity leads not only to increased fat depots in classical adipose tissues locations but also to significant lipid deposits within and around other tissues and organs, a phenomenon known as ectopic fat storage. The key target-organs include primarily the heart, blood vessels and kidney (2).

Novel cardiovascular risk biomarkers such as UACR, hsCRP, and NT-proBNP have all been observed to predict cardiovascular disease in the general population. UACR, 
hsCRP and NT-proBNP provide additive prognostic information in the general population as well as hypertensive population, perhaps because they are biomarkers of damage in different parts of the cardiovascular system. High UACR represents microvascular damage, high hsCRP represents atherosclerosis, while high NT-proBNP represents cardiovascular hypertrophy and left ventricular dysfunction. The additive effects of these three risk markers could also reflect that these damaging processes do not occur simultaneously in the cardiovascular system. Early atherosclerosis could be the initial event followed by microvascular damage and subsequently cardiovascular hypertrophy and left ventricular dysfunction (3).

Cardiovascular disease is primarily caused by atherosclerosis, the condition begins with endothelial dysfunction until intima blockage occurs in artery blood vessels, promoting reduction of elasticity, structure and decreased blood flow. Atherosclerosis in blood vessel leads to coronary heart disease. Atherosclerosis constitutes aggregate of foam cells, derived from macrophage containing lipid. Accumulated cholesterol in foam cells is from lipid circulation, primarily oxidized LDL resulted from vascular inflammation and oxidative stress $(4,5)$.

LDL is modified by oxidation to produce an oxidized form of LDL (oxLDL), which promotes atherosclerosis. OxLDL promotes foam cells formation and attracts monocytes, lymphocytes and macrophages, while causing vasoconstriction and cells proliferation. These effects, coupled with increased apoptosis and plaque rupture, induction of pro-inflammatory genes, and antigenicity, lead to atherogenesis (6). So, in this study we used OxLDL as a marker of atherosclerosis instead of Doppler Ultrasound, which is more time consuming and expensive.

Based on the above backgrounds, we consider that this study on the correlation of UACR, hsCRP and NTproBNP with the development of atherosclerosis in centrally obese men was necessary to carry out.

\section{Methods}

This study was observational with cross-sectional design done on a group of centrally obese men $(n=76)$ in Bandung and Jakarta. The subjects' ages were between 30-50 years, they had central obesity with waist circumference $\geq 90$ $\mathrm{cm}$. Before commencement of the study, all subjects signed an informed consent. None of the study subjects had acute inflammation (hSCRP $\geq 10 \mathrm{mg} / \mathrm{dL}$ ), diabetes mellitus, kidney disorder, urinary tract infection, heart failure, severe hypertension, high fever, consumption of anti-inflammation or antioxidant supplements. All subjects underwent standardized interviews, physical and laboratory examinations namely urine, fasting blood glucose, blood creatinine, UACR, hsCRP, NT-proBNP and OxLDL.

Urine albumin was assessed by immunoturbidimetric assay (Roche, number 03576108190 ) and urine creatinine was determined by Jaffe without deproteinase (Roche, number 1875418). hsCRP by chemiluminescent immunometric assay (Siemens, number LKCRP2), NTproBNP by electrochemiluminescence (ECLIA) (Roche, number 04917049) and OxLDL by ELISA (Mercodia, number 10114301).

Statistical analysis was done by SPSS for Windows v 13.0 with a significance level at $\mathrm{p}<0.05$. The correlations between biomarkers were assessed by Pearson or Spearman's rho correlation.

\section{Results}

Table 1 and Table 2 provide information on the general and metabolic characteristics of the study subjects. On each subject characteristic we did normal distribution test (Kolmogorov Smirnov test). From the test some parameters were found of no normal distribution $(\mathrm{p}<0.05)$ viz weight, body mass index, waist circumference, systolic blood pressure, diastolic blood pressure, UACR and NT-proBNP. For normalization of the distribution, we then applied logarithm adjustment for these parameters. Of these 76 subjects, based on the body weight and height, $23.7 \%$ had BMI $<25 \mathrm{~kg} / \mathrm{m}^{2}$ and $76.3 \%$ had BMI $\geq 25 \mathrm{~kg} / \mathrm{m}^{2}$.

To evaluate correlation of body mass index and waist circumference for each parameter, was done correlation test between each marker. Table 3 shows results of the correlation test.

To evaluate correlation of UACR, hsCRP and NTproBNP with OxLDL was done correlation test between each marker. Table 4 shows results of the correlation test. From the correlation test, we found a significant linear positive correlation between OxLDL and hsCRP. 
Table 1. General Characteristics of Study Subjects

\begin{tabular}{lrrrrr}
\hline Characteristics & Mean & SD & Minimum & Maximum & $p$ \\
\hline Age $($ year) & 37.00 & 5.93 & 30.00 & 49.00 & 0.119 \\
Weight $(\mathrm{kg})$ & 78.90 & 11.83 & 62.50 & 121.00 & 0.013 \\
Height $(\mathrm{cm})$ & 168.81 & 5.92 & 158.00 & 185.00 & 0.127 \\
BMl $\left(\mathrm{kg} / \mathrm{m}^{2}\right)$ & 27.68 & 3.79 & 22.30 & 40.40 & 0.018 \\
WC $(\mathrm{cm})$ & 98.34 & 7.24 & 90.00 & 150.00 & 0.015 \\
SBP $(\mathrm{mmHg})$ & 116.00 & 111.15 & 90.00 & 150.00 & 0.002 \\
DBP $(\mathrm{mmHg})$ & 77.00 & 7.85 & 60.00 & 90.00 & 0.000 \\
\hline
\end{tabular}

$\mathrm{BMI}=$ Body Mass Index $; \mathrm{WC}=$ Waist Circumference, $\mathrm{SBP}=$ Systolic Blood Pressure, DBP $=$ Diastolic Blood Pressure

Table 2. Metabolic Characteristics of Study Subjects

\begin{tabular}{lrrrrr}
\hline Characteristics & Mean & SD & Minimum & Maximum & p \\
\hline Fasting Blood Glucose (mg/dL) & 93.17 & 9.13 & 70.00 & 121.00 & 0.438 \\
Creatinine (mg/dL) & 0.91 & 0.12 & 0.60 & 1.20 & 0.053 \\
UACR ( $\mu$ g/mg kreat) & 8.01 & 7.78 & 3.00 & 43.00 & 0.000 \\
hsCRP (mg/L) & 2.38 & 1.96 & 0.31 & 8.59 & 0.063 \\
NT-proBNP (pg/mL) & 23.43 & 13.27 & 7.00 & 75.38 & 0.007 \\
OxLDL (U/L) & 69.55 & 24.92 & 30.20 & 133.50 & 0.068 \\
\hline
\end{tabular}

UACR = Urine Albumin Creatinine Ratio, hs CRP = high sensitivity C-Reactive Protein, NT-proBNP = N-Terminal Pro Brain Natriuretic Peptide, OxLDL = Oxidized Low Density Lipoprotein.

Table 3. Correlation of UACR, hsCRP and NT-proBNP with BMI and WC

\begin{tabular}{lccccc}
\hline Parameters & & UACR & hsCRP & Log NT-proBNP & OxLDL \\
\hline \multirow{2}{*}{ Log BMI Correlation } & $(\mathrm{r})$ & 0.266 & 0.223 & -0.126 & 0.028 \\
& $(\mathrm{p})$ & 0.020 & 0.053 & 0.277 & 0.809 \\
\multirow{2}{*}{ WC correlation } & $(\mathrm{r})$ & 0.274 & 0.111 & -0.085 & -0.165 \\
& $(\mathrm{p})$ & 0.017 & 0.338 & 0.463 & 0.153 \\
\hline
\end{tabular}

Table 4. Correlation of UACR, hsCRP and NT-proBNP with OxLDL

\begin{tabular}{lcccc}
\hline Parameters & & UACR & hsCRP & Log NT-proBNP \\
\hline OxLDL Correlation & (r) & -0.138 & 0.230 & -0.173 \\
& (p) & 0.236 & 0.046 & 0.136 \\
\hline
\end{tabular}




\section{Discussion}

\section{Analysis of Correlation of Obesity, Urine Albumin/ Creatinine Ratio (Endothelial Dysfunction) with} OxLDL (Atherosclerosis)

In this study we found that UACR had significant linear correlation with BMI and waist circumference as evidenced by $p=0.020$ and $p=0.017$. This finding agreed with that of the study by Tamba, et al. on Japanese subjects, where $\log$ UACR corelated with $\log$ BMI and $\log$ WC corelated with $\mathrm{p}=0.0001$ and $\mathrm{p}<0.0001$. The study also showed that visceral lipid acummulation was related with the increase of UACR result. It was also mentioned that the subjects who had visceral lipid $\geq 100$ $\mathrm{cm}^{2}$ consistently had high UACR result. Measurement of visceral lipid used impedance bioelectrical analyzing method (7). However, in our present study we did not do visceral lipid assessment.

A controversy still exists about the correlation between visceral lipid and urine albumin. It's not yet very clear whether acummulation of visceral lipid alone or together with the other risk factors such as hypertension, hyperglycemia or dyslipidemia can be associated with the increase of urine albumin excretion. Tamba et al. have shown in their study that visceral lipid acummulation correlated with the increase of urine albumin as an independent risk factor. Albuminuria is a condition that results from gromerular leakage wherein albumin can pass through the glomerular filtratrion barrier until albumin is detected in the urine. Urine albumin/creatinine ratio can be used as a common endothelial dysfunction marker to see if albuminuria exists (8).

Albuminuria is not only used as an indicator for existence of glomerular damage, but it is also a sensitive indicator for detecting endothelial dysfunction or capillary vasculopathy that can cause lipoprotein flow through the arterial wall. Although albuminuria is known to be associated with endothelial dysfunction of the circulatory system, the true mechanism of the association is not yet very clear.

Theoretically, endothelial dysfunction contributes directly to the pathogenesis of albuminuria that causes increase of gromerular pressure and gromerular leakage, and indirectly affects the gromerular messangial and epithelial cell function (9).

On Steno hypothesized that albumin leakage into urine was a reflection of existing vascular damage. The hypothesis related the condition of endothelial dysfunction with albumin leakage in vascular sites (10).
In this study we found no significant correlation between UACR and atherosclerosis, as evidenced by OxLDL $p=0.236$ showing a tendency of negative correlation $r=-0.138$. Our study results showed only three subjects had UACR $>30 \mu \mathrm{g} / \mathrm{mg}$. We know that visceral lipid acummulation causes greater oxidative stress production, and that increased oxidative stress can further cause dysregulation of adipocytokine production in the form of decreased adiponectin serum that finally causes increase of urine albumin (8).

\section{Analysis of Correlation between Obesity, hsCRP (Inflammation) and OxLDL (Atherosclerosis)}

This study showed that waist circumference and body mass index had a linear correlation with inflamation (hsCRP), although not significantly $(\mathrm{p}=0.053)$. In the categorial analysis we found that there was a significant correlation between BMI and hsCRP, with $\mathrm{p}=0.012, \mathrm{OR}=3.929$. It can be concluded that among the group of subjects having BMI $\geq 25 \mathrm{~kg} / \mathrm{m}^{2}$ more subjects with hsCRP $\geq 1 \mathrm{mg} / \mathrm{L}$ could be found as compared with other group having BMI $<25 \mathrm{~kg} / \mathrm{m}^{2}$.

In central obesity, adipocytes are stimulated to synthesize proinflammatory adipokines/cytokines such as TNF- $\alpha$ and Interleukin-6. TNF- $\alpha$ highly increases in obesity that can stimulate CRP production. TNF- $\alpha$ induces synthesis of Interleukin- 6 that constitutes the main regulator of CRP synthesis. Interleukin-6 stimulates the liver to produce CRP as vascular biomarker. The level of hsCRP can be regarded as an atherosclerosis predictor, with value ranges $<1 \mathrm{mg} / \mathrm{L}$ for low risk, 1-3 $\mathrm{mg} / \mathrm{L}$ for moderate risk, and $3-10 \mathrm{mg} / \mathrm{L}$ for high risk of cardiovascular disease (11).

In this study we found weak correlation significance between obesity and inflammation, thus we assumed that the inflammation process in the study subjects had not lasted for a very long time yet. The subjects involved in this study were at the average age of 37 years, so they were not yet posed to significant hsCRP enhancement.

According to the theory, atherosclerosis starts with inflammation in the endothelial that causes expression of adhesion molecules. Adhesion molecules attract monocytes which then migrate through the endothelial layer because of various proinflammatory chemoattractants. Inside the intima, monocytes continue undergoing alteration to inflammation and differentiation of macrophages to phagocyte lipid to become foam cells. Lymphocyte $\mathrm{T}$ also migrate inside the intima, unattached from proinflammatory cytokine that reduplicates inflammatory activity. Through that inflammation process, the early lesion of atherosclerosis contains formed lipid 
layer. Inflammation also has a role in complex plaque development and formation of weak fibrous layer, which may then cause plaque rupture and eventually increased trombogenicity lipid layer (12).

\section{Analysis of Correlation of Obesity and NT-proBNP with OxLDL (Atherosclerosis)}

In this study, it was shown that waist circumference and body mass index had no significant correlation, but the former with NT-proBNP showed the tendency of negative correlation. From the categorial analysis it was found that more individuals among the subjects with BMI $\geq 25 \mathrm{~kg}$ / $\mathrm{m}^{2}$ had higher value of NT-proBNP compared to subjects with BMI $<25 \mathrm{~kg} / \mathrm{m}^{2}$.

An earlier study by Fernandes has shown that increase of NT-proBNP correlated with the duration of obesity; of which the average obesity duration was 15,64 years (13). Data of obesity duration in our study were unfortunately not recorded. The longer obesity duration with increase of body mass index is known as a risk factor for left ventricle remodelling and heart failure events because non adipose cells have a limited capacity to store lipid (14).

Other earlier studies have observed that in decreased body mass index there was increased plasma NT-proBNP level suggesting increased death risk in CHF patients. Obesity is associated with salt retention and increased cardiac output, which would be expected to produce elevated natriuretic peptide levels. Natriuretic peptide clearance receptors (NPR-C) are abundant in adipose tissue, suggesting that adipocytes participate in removal of natriuretic peptides from the circulation. In experimental animals, caloric depriviation through fasting results in dramatic decreases in NPR-C gene expression and increased circulating ANP levels. Elevated NPR-C gene expression has been documented in the adipose tissue of humans with obesity and hypertension, and allelic variants of this gene have been associated with lower plasma natriuretic peptide levels (15).

An experimental study on rodents has shown more lipid accumulation in the heart muscle wall presented cardiotoxic property, which also caused left ventricular hypertrophy and widening of non-systemic cardiomyopathy (16). Left ventricle hypertrophy is an independent risk factor for subclinical atherosclerosis and also related with inflammatory condition as shown by the increase in CRP value, although this relation seems to appear only in abnormal condition (17).

Parallel to the relationship of body mass index and waist circumference with NT-proBNP, correlation between NT-proBNP and OxLDL in this study showed no significant correlation. Thickness of the ventricle wall correlates with atherosclerosis, but the basic mechanism of its development and that of left ventricle hypertrophy is not yet known. In this study, the subjects studied had not yet had significant left ventricle remodelling, so no significant increase of NT-proBNP level was encountered.

\section{Conclusion}

Occurrence of atherosclerosis was significantly correlated with hsCRP (low grade inflammation).

\section{Acknowledgement:}

The authors thank the Prodia Education and Research Institute for their support for this study.

\section{References:}

1. International Diabetes Federation. Consensus on the Metabolic Syndrome: Definition and Treatment. Berlin. 2005

2. Montani JP, Carroll JF, Dwyer TM, Antic V, Dulloo AG. Ectopic Fat Storage in Heart, Blood Vessels and Kidneys in the Pathogenesis of Cardiovascular Disease. Int J Obes 2004; 28: S58-65

3. Olsen MH, Hansen TW, Christensen MK, Gustafsson F, Rasmussen S, Wachtell $\mathrm{K}$, et al. Cardiovascular Risk Prediction by N-Terminal Pro Brain Natriuretic Peptide and High Sensitivity C-Reactive Protein is Affected by Age and Sex. J Hypertens 2007; 26: 26-34.

4. Steinberg D. Low Density Lipoprotein Oxidation and Its Pathobiological Significance. J Bio Chem 1997; 34: 20963-6.

5. Heinecke JW. Mechanisms of Oxidative Damage of Low Density Lipoprotein in Human Atherosclerosis. Curr Opin Lipidol 1997; 8: 268-74.

6. Koenig W, Khuseyinova N. Biomarkers of Atherosclerotic Plaque Instability and Rupture. Arterioscler Thromb Vasc Biol 2007; 27: 15-26.

7. Tamba S, Nakatsuji H, Kishida K, Noguchi M, Ogawa T, Okauchi $\mathrm{Y}$, et al. Relationship between Visceral FatAccumulation and Urine Albumin-Creatinine Ratio in Middle-Aged Japanese Men. Atherosclerosis 2010; 211: 601-5.

8. Furukawa S, Fujita T, Shimabukuro M, Iwaki M, Yukio Y, Nakajima Y, et al. Increased Oxidative Stress in Obesity and its Impact on Metabolic Syndrome. J. Clin. Invest 2004; 114: 1752-61.

9. Satchell SC, Tooke JE. What is the Mechanism Of Microalbuminuria in Diabetes: A Role for the Glomerular Endothelium?. Diabetologia 2008; 51: 714-25.

10. Weir MR. Microalbuminuria and Cardiovascular Disease. Clin J Am Soc Nephrol 2007; 2: 581-90.

11. Lyon CJ, LawRE, Hsueh WA. Minireview:Adiposity, Inflammation, and Atherogenesis. Endocrinol 2003; 144: 2195-200.

12. Libby P. Inflammation and Cardiovascular Disease Mechanisms. Am J Clin Nutr 2006; 83: 456S-60S.

13. Fernandes F, Ramires FJA, Buck PC, Almeida IJ, Rabelo R, Dantas SA, et al. N-Terminal-Pro-Brain Natriuretic Peptide, 
but not Brain Natriuretic Peptide, is Increased in Patients with Severe Obesity. Braz J Med Bio Res 2007; 40: 153-8.

14. Szczepaniak LS, Victor RG, Orci L, Unger RH. Forgotten but not Gone. The Rediscovery of Fatty Heart, the Most Common Unrecognized Disease in America. Circ Res 2007; 101: 759-67.

15. Wang TJ, Larson MG, Levy D, Benjamin EJ, Leip EP, Wilson PWF, et al. Impact of Obesity on Plasma Natriuretic Peptide
Levels. J Am Soc Nephrol 2004; 109: 594-600.

16. McGavock MJ, Victor RG, Unger RH, Szczepaniak LS. Adiposity of the Heart. Ann Intern Med 2006; 144: 517-24.

17. Mehta SK, Rame E, Khera A, Murphy AA, Canham RM, Peshock RM, et al. Left Ventricular Hypertrophy, Subclinical Atherosclerosis and Inflammation. Hypertension 2007; 49: 1385-91. 